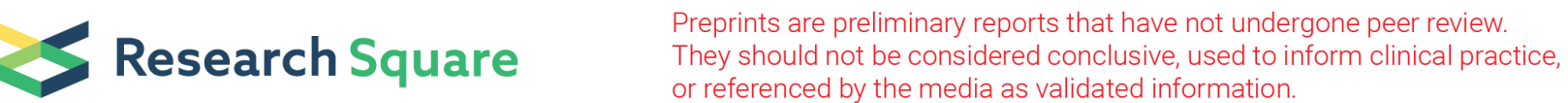

\section{An In-Silico Analysis of Differentially Expressed Genes and mi-RNA Regulating Them in HPV Positive and HPV Negative Head and Neck Cancers (H\&NC).}

Jyoti Shekhawat

All India Institute of Medical Sciences Jodhpur Ashita Gadwal

All India Institute of Medical Sciences Jodhpur Manoj Khokhar

All India Institute of Medical Sciences Jodhpur Kavya Gauba

All India Institute of Medical Sciences Jodhpur Shruti Gupta

All India Institute of Medical Sciences Jodhpur

Bikram Choudhury

All India Institute of Medical Sciences Jodhpur

Mithu Banerjee ( $\triangle$ mithu.banerjee.3@gmail.com )

All India Institute of Medical Sciences Jodphur https://orcid.org/0000-0002-4107-3076

\section{Research Article}

Keywords: Head and neck cancers, Human papillomavirus, CAV1, microarray

Posted Date: May 28th, 2021

DOI: https://doi.org/10.21203/rs.3.rs-505637/v1

License: (c) (i) This work is licensed under a Creative Commons Attribution 4.0 International License. Read Full License 


\section{Abstract}

HPV contributes to the pathogenesis of H\&NC. H\&NC patients are diagnosed at an advanced stage and are more disposed to lymph node metastasis. There is conflicting evidence with regard to distant metastasis in H\&NC. Identification of potential candidate genes which can be used as predictors of prognosis in HPV positive/negative H\&NCs are needed. We initiated the study using publicly available microarray gene expression datasets from the GEO. Functional enrichment analysis of significant genes was done using the DAVID. The PPI network was constructed in the STRING and visualized using Cytoscape. The CytoHubb plugin was utilized to identify the key genes in this complicated network. The MCODE plugin was applied to screen out significant gene modules. Hub genes identified were analysed for overall survival (OS) in GEPIA. Analysis of the miRNA and transcription factors targeting the hub gene with significance was done using miRNet. GEO datasets GSE39366, GSE40774, GSE117973 were used in this study. We identified 67 overlapping DEGs which were significant. Gene Ontology identified key terms as 'cellular component', 'biological process', and 'molecular function' respectively. Cytoscape and MCODE revealed two significant modules. The common hub genes identified using cytohubb plugin. CAV1 has been reported to be significantly associated with OS in H\&NC patients. 3 targeting miRNA and 54 transcription factors were identified using miRNet. In-silico survival and expression analyses revealed CAV1 as a candidate gene that may be used to predict the course of disease progression in HPV positive and HPV negative H\&NC patients. Further invitro studies validating CAV1 and its three target miRNAs as predictors of prognosis in $\mathrm{H} \& \mathrm{NC}$ are highly warranted.

\section{Introduction:}

Head and neck cancers are the sixth leading cancer worldwide with approximately 550,000 new cases and about 300,000 deaths every year (HeadNeck.Pdf, n.d.). Head and neck cancers arise in the epithelial lining of the nasopharynx, larynx, oropharynx, oral cavity. The major etiological factors of H\&NCs are human papillomavirus (HPV), tobacco products and alcohol consumption. HPV 16 and HPV 18 are major subtypes that cause infection. HPV infection is most commonly present in the oropharynx and oral cavity (McKaig et al., 1998). H\&NC can be categorized into two categories based on HPV status. HPV positive $\mathrm{H} \& \mathrm{NC}$ occur due to HPV infection while the association of alcohol, tobacco and alcohol and tobacco both in HPV negative H\&NC is more pronounced (Auguste et al., 2020). Although, there are several treatment modalities $\mathrm{H} \& \mathrm{NC}$ are mostly diagnosed at an advanced stage with lower 5 -year survival rates. It has been earlier established that HPV positive H\&NC have a better prognosis compared to HPV negative. HPV positive patients show a better response to radiotherapy and chemotherapy (Elrefaey et al., 2014).

Several studies have used microarray-based analysis to identify the differentially expressed genes (DEGs) in H\&NC (Kuriakose et al., 2004). Thus, there is a need to identify potential candidate genes which can be used to track the disease progression in HPV positive/negative H\&NCs. Therefore, in the present study, we aimed to identify the DEGs between HPV positive and HPV negative H\&NC, through publicly available microarray gene expression datasets from the Gene Expression Omnibus (GEO). Using an in-silico approach, Kaplan-Meier survival analysis of hub genes was performed to identify the hub genes involved 
in the prognosis of H\&NC. Further, mi-RNA and transcription factors specific to hub genes which were significantly associated with poor prognosis of H\&NC were also identified.

\section{Materials And Methods:}

2.1 Microarray datasets: The microarray data were acquired from the gene expression omnibus (GEO) database (https://www.ncbi.nlm.nih.gov/geo/). Three gene expression datasets (GSE39366, GSE40774, GSE117973) of H\&NC were included in this study. The detailed information of each dataset microarray was summarized in Table 1.

2.2 Identification of differentially expressed genes (DEGs): The interactive web tool GEO2R (https://www.ncbi.nlm.nih.gov/geo/geo2r/) was used to screen DEGs between HPV positive samples and HPV negative samples in H\&NC. P-value $<0.05$ and $|\log F C| \geq 1.0$ were set as the threshold for DEGs. Overlapping DEGs were identified using the Venn bioinformatics tool (http://bioinformatics.psb.ugent.be/webtools/Venn/) (Draw Venn Diagram, n.d.).

2.3 Functional enrichment analysis of DEGs: Gene ontology (GO) describes the genes and gene products into three categories namely biological processes (BP), molecular functions (MF), and cellular components (CC) (Gene Ontology Consortium, 2006). Kyoto Encyclopedia of Genes and Genomes (KEGG) is a database for the analysis and linkage of genes to higher-level systemic functions of a given cell, organism, or ecosystem (Kanehisa \& Goto, 2000). GO and KEGG were analyzed with the Database for Annotation, Visualization and Integrated Discovery (DAVID) (https://david.ncifcrf.gov/). The cutoff criterion was set at $\mathrm{P}<0.05$.

2.4 Protein-protein interaction (PPI) network construction: The PPI network of DEGs was identified using the search tool for the retrieval of interacting genes (STRING) database (https://string-db.org/) (Szklarczyk et al., 2017). Subsequently, Cytoscape software v3.8.2 was used to visualize the PPI network (https://cytoscape.org/) (Shannon et al., 2003).

2.5 Hub gene identification and module analysis from the PPI network: The CytoHubb plugin was utilized to identify the key genes in this complicated network. The top 10 genes with the highest scores were determined as hub genes and subjected to further analyses. The Molecular Complex Detection (MCODE) plugin was applied to screen out significant gene modules. The most significant modules in the PPI network were identified by the Molecular Complex Detection (MCODE) plugin with MCODE scores $\geq 3$, degree cut-off $=2$, node score cut-off $=0.2$, Max depth $=100$ and $\mathrm{k}$-core $=2$.

2.6 Survival analysis of hub genes: Gene Expression Profiling Interactive Analysis (http://gepia.cancerpku.cn/) is an online tool used to predict patient survival based on gene status. The genes of interest were entered into the tool. The patients were divided into two groups based on their gene expression levels, and their survival rates (Kaplan Meier) were analyzed. The $95 \%$ confidence intervals and log-rank P-values of the hazard ratios were calculated. 
2.7 Identification of potential transcription factors and mi-RNA regulating hub genes: Hub gene targeting miRNAs were predicted by using miRNet 2.0 database (https://www.mirnet.ca/) a miRNA-centric network visual analytics platform, and hub gene targeting transcription factors were analysed using miRNet at four different platforms (ENCODE, JASPAR, REGNETWOTK and TRUST). Further, hub gene-miRNA and hub gene transcription factor interaction networks were visualized in Cytoscape.

Table 1

Details of the GEO datasets.

\begin{tabular}{|lllll|}
\hline Reference & GEO & Platform & HPV-positive & HPV-negative \\
\hline Walter et.al (6) & GSE39366 & GPL9053 & 14 & 82 \\
\hline Keck et.al (7) & GSE40774 & GPL13497 & 58 & 76 \\
\hline Schmitt et.al (8) & GSE117973 & GPL10558 & 24 & 53 \\
\hline
\end{tabular}

\section{Results:}

3.1 Identification of DEGs: The gene expression datasets GSE39366, GSE117973 and GSE40774 were acquired from the GEO database. DEGs between HPV-positive and HPV-negative samples were screened using GEO2R. As a result, 337, 403 and 444 DEGs were identified from the GSE39366, GSE117973 and GSE40774 datasets, respectively. Volcano plots were generated for all the datasets for the intuitive representation of the DEGs Fig. 1(a-c). The blue plots represent downregulated DEGs, the red plots represent upregulated DEGs, and the black plots are not DEGs. Besides, Venn diagrams were also drawn for comparison of the number of total DEGs, upregulated DEGs, and downregulated DEGs in the three datasets. As a result, 67 DEGs were identified, comprised of 40 up-regulated and 27 down-regulated DEGs shown in Fig. 1(d).

3.2 Functional enrichment of DEGs: KEGG pathway and GO terms for DEGs were analyzed using David shown in Fig. 2(a-d). The top biological process in which identified DEGs were enriched includes epidermis development, cytoskeleton organization, keratinocyte differentiation, positive regulation of positive chemotaxis, positive regulation of cell migration, $\mathrm{G} 1 / \mathrm{S}$ transition of the mitotic cell cycle, cell adhesion. The cellular components were enriched in extracellular exosome, intermediate filament whereas molecular functions were enriched in the structural constituent of cytoskeleton, protein kinase binding. The KEGG pathways were enriched in cell cycle and arachidonic acid metabolism.

3.3 Construction of the PPI network and module analysis: To further explore the interaction between 67 common DEGs, a PPI network was constructed shown in Fig. 3(a). The PPI network contained 41 nodes and 57 edges. 2 significant modules were obtained from the PPI network using the MCODE plugin. The first module consisted of KRT6B, KRT15, KRT16, KRT19 and KRT75 and the second module had STAG3, YBX2 a ZCWPW1 shown in Fig. 3(b) and 3(c). The hub genes were identified using MCC, DMNC, EPC, and Degree algorithms in the Cytohubba plugin. The common hub genes (SPRR1B, CAV1, KRT16, KRT6B, KRT15, KRT19 \& KRT75) were identified by the Venn diagram highlighted in Fig. 3(a) with red colour. 
3.4 Survival analysis of Hub genes: Association between hub genes and overall survival (OS) of head and neck cancer patients were analyzed. Only CAV1 $(p=0.0016, H R=1.5)$ showed a significant association with OS shown in Fig. 4(a-b).

3.5 Transcription factors and mi-RNA regulating hub genes: Hub gene targeting miRNA were predicted using miRNet, based on the correlation analysis between the hub gene and miRNAs. The network consisted of one gene, 54 transcription factors and 3 mi-RNAs shown in Fig. 4(c).

\section{Discussion:}

Head and neck cancer (H\&NC) are among the most common cancers with a poor prognosis. The human papillomavirus is known as an oncogenic driver in H\&NC. HPV has many subtypes but the most common subtypes responsible for the infection are HPV 16 and HPV 18. HPV mostly infects the oral cavity and oropharyngeal regions. The pathogenesis of human papillomavirus (HPV)-positive and HPV-negative HNSCCs differs. H\&NC subtypes based on HPV status show different clinicopathological features. HPV positive patients show early lymph node metastasis with disease progression (Jensen et al., 2014). However, Dok et.al propose that the rate of distant metastasis is equal in both HPV positive and HPV negative H\&NC(Dok et al., 2017). There is conflicting evidence regarding distant metastasis in HPV positive and HPV negative H\&NC. Only a handful of studies have considered the HPV status when identifying biomarkers to predict the course of H\&NC. Thus, the identification of genes-specific to HPVpositive and HPV-negative H\&NC is urgently needed which can be used as potential biomarkers to track disease progression.

Caveolins are major structural proteins found in caveolae (a special type of lipid rafts, small invaginations of plasma membrane present in endothelial cells, adipocytes), consist of CAV1, CAV2 \& CAV3. These proteins are highly expressed in terminally differentiated cells- adipocytes, endothelial cells, fibroblasts. CAV1 is a $22 \mathrm{kDa}$ protein that is the principal substrate of src kinase (Glenney \& Zokas, 1989) and appears as a filament-like structure at the plasma membrane (Rothberg et al., 1992). CAV1 acts as double-edged sword. It functions both as tumor suppressor gene as well as an oncogene (Chanvorachote \& Chunhacha, 2013; Huang et al., 2012, p. 1). Tumor suppressive role of CAV1 is observed in early stages of cancer where its downregulation is associated with tumour progression however, at the advanced stages its overexpression correlates with metastasis, lymph node involvement (Arpaia et al., 2012; Nestl et al., 2001; Tse et al., 2012). The oncogenic role of CAV1 has been identified in many cancer types including breast, prostate, oesophagal. It has been associated with carcinogenesis including cellular transformation, tumour growth, cell migration and metastasis, epithelial to mesenchymal transition, cell death and survival, multidrug resistance (MDR) and angiogenesis.

A cDNA microarray-based study has shown decreased expression of CAV1 in HPV positive H\&NC and cervical cancers compared to HPV negative H\&NC (Longworth et al., 2005, p. 2). In HNSCC overexpression of CAV1 was found to be associated with concurrent abnormal expression of at least one member of the E-cadherin/ $a-\beta$ catenin complex and multiple ErbB receptors as well as with lymph node 
metastases (Masuelli et al., 2012). CAV1 and CAV2 are also found to be overexpressed in the oesophagal squamous cell carcinoma patients where it was related with shorter survival and as a prognostic marker (Ando et al., 2007). In hypopharyngeal squamous cell carcinoma overexpressed levels of CAV1 were associated with clinical parameters (TNN stage, tumour grade and lymph node metastasis) (Zhao et al., 2016). CAV1 plays a critical role in metastasis of HNSCC via the EMT process and the formation of stem cells (Masood et al., 2013). This has been elucidated on prostate cancer patients where elevated expression of CAV1 was positively correlated to gleason score, lymph node involvement and positive surgical margin (Yang et al., 1999). Kato et.al have shown that overexpression of CAV1 in oesophageal SCC was associated with lymph node metastasis and worse progression after surgery (Kato et al., 2002, p. 1).

Anoikis, a form of programmed cell death induced in cells when they are detached from extracellular matrix, is a hallmark of cancer progression towards metastasis. Low expression of CAV1 has been shown in patients with lymph node metastasis as well as in in-vivo mouse models compared to the primary tumour with or without lymph node involvement. CAV1 disrupts integrin 31 -src oncogene interaction to inhibit tumour formation and metastasis and moves the cell towards the anoikis process (Zhang et al., 2008). In addition to this, low or complete disappearance of CAV1 in H\&NC patients has shown EMT and prometastatic properties (Jung et al., 2015).

Hypermethylation of CAV1 promoter was associated with lymph node metastasis in breast cancer patients and it also correlated with decreased disease-free survival (Alevizos et al., 2014). Also, the treatment of MCF-7 and MDA-MB-231 breast cancer cell lines with 5-aza have increases the expression of CAV1 mRNA and protein levels (Deb et al., 2014). Similar results were seen in HNSCC metastatic cell line on treatment with 5-aza-dc. CAV 1 level was restored which is similar to that expressed in parental OSCC $686 \mathrm{LN}$ cell (Zhang et al., 2008).

miR-133a is a tumour suppressor miRNA. A bioinformatics based approach has identified CAV1 as one of its targets in HNSCC (Nohata, Hanazawa, Kikkawa, Mutallip, Fuse, et al., 2011). miR-133a binds at the CAV1 mRNA sequence. CAV1 expression was decreased on transfection with miR-133a in HSC3 and SAS HNSCC cell lines (Nohata, Hanazawa, Kikkawa, Mutallip, Fujimura, et al., 2011). In this study, we identified that CAV1 is regulated by miR-7-5p, miR-145-5p and miR200b-3p. Garrido et.al have also demonstrated that miR-145-5p regulates the CAV1 expression in epithelial ovarian cancer (Garrido et al., 2020).

Our study identified CAV1 as a potential oncogene-specific to HPV status of H\&NC. CAV1 has been identified as a potential gene involved in lymph node metastasis in H\&NC. Decrease in CAV1 expression correlates with progression of disease. CAV1 may be used as a candidate gene to predict prognosis of HPV positive patients. In early stage patients use of recombinant CAV1 may serve as potential therapeutic option.

\section{References}


Alevizos, L., Kataki, A., Derventzi, A., Gomatos, I., Loutraris, C., Gloustianou, G., Manouras, A., Konstadoulakis, M. M., \& Zografos, G. (2014). Breast cancer nodal metastasis correlates with tumour and lymph node methylation profiles of Caveolin-1 and CXCR4. Clinical \& Experimental Metastasis, 31(5), 511-520. https://doi.org/10.1007/s10585-014-9645-6

Ando, T., Ishiguro, H., Kimura, M., Mitsui, A., Mori, Y., Sugito, N., Tomoda, K., Mori, R., Harada, K., Katada, T., Ogawa, R., Fujii, Y., \& Kuwabara, Y. (2007). The overexpression of caveolin-1 and caveolin-2 correlates with a poor prognosis and tumor progression in esophageal squamous cell carcinoma. Oncology Reports, 18(3), 601-609. https://doi.org/10.3892/or.18.3.601

Arpaia, E., Blaser, H., Quintela-Fandino, M., Duncan, G., Leong, H. S., Ablack, A., Nambiar, S. C., Lind, E. F., Silvester, J., Fleming, C. K., Rufini, A., Tusche, M. W., Brüstle, A., Ohashi, P. S., Lewis, J. D., \& Mak, T. W. (2012). The interaction between caveolin-1 and Rho-GTPases promotes metastasis by controlling the expression of alpha5-integrin and the activation of Src, Ras and Erk. Oncogene, 31(7), 884-896. https://doi.org/10.1038/onc.2011.288

Auguste, A., Deloumeaux, J., Joachim, C., Gaete, S., Michineau, L., Herrmann-Storck, C., Duflo, S., \& Luce, D. (2020). Joint effect of tobacco, alcohol, and oral HPV infection on head and neck cancer risk in the French West Indies. Cancer Medicine, 9(18), 6854-6863. https://doi.org/10.1002/cam4.3327

Chanvorachote, P., \& Chunhacha, P. (2013). Caveolin-1 Regulates Endothelial Adhesion of Lung Cancer Cells via Reactive Oxygen Species-Dependent Mechanism. PLOS ONE, 8(2), e57466.

https://doi.org/10.1371/journal.pone.0057466

Deb, M., Sengupta, D., Kar, S., Rath, S. K., Parbin, S., Shilpi, A., Roy, S., Das, G., \& Patra, S. K. (2014). Elucidation of caveolin 1 both as a tumor suppressor and metastasis promoter in light of epigenetic modulators. Tumour Biology: The Journal of the International Society for Oncodevelopmental Biology and Medicine, 35(12), 12031-12047. https://doi.org/10.1007/s13277-014-2502-z

Dok, R., Glorieux, M., Holacka, K., Bamps, M., \& Nuyts, S. (2017). Dual role for p16 in the metastasis process of HPV positive head and neck cancers. Molecular Cancer, 16(1), 113. https://doi.org/10.1186/s12943-017-0678-8

Draw Venn Diagram. (n.d.). Retrieved January 15, 2021, from http://bioinformatics.psb.ugent.be/webtools/Venn/

Elrefaey, S., Massaro, M. A., Chiocca, S., Chiesa, F., \& Ansarin, M. (2014). HPV in oropharyngeal cancer: The basics to know in clinical practice. Acta Otorhinolaryngologica Italica: Organo Ufficiale Della Societa Italiana Di Otorinolaringologia E Chirurgia Cervico-Facciale, 34(5), 299-309.

Garrido, M. P., Torres, I., Avila, A., Chnaiderman, J., Valenzuela-Valderrama, M., Aramburo, J., Oróstica, L., Durán-Jara, E., Lobos-Gonzalez, L., \& Romero, C. (2020). NGF/TRKA Decrease miR-145-5p Levels in 
Epithelial Ovarian Cancer Cells. International Journal of Molecular Sciences, 21(20), 7657. https://doi.org/10.3390/ijms21207657

Gene Ontology Consortium. (2006). The Gene Ontology (GO) project in 2006. Nucleic Acids Research, 34(Database issue), D322-326. https://doi.org/10.1093/nar/gkj021

Glenney, J. R., Jr, \& Zokas, L. (1989). Novel tyrosine kinase substrates from Rous sarcoma virustransformed cells are present in the membrane skeleton. Journal of Cell Biology, 108(6), 2401-2408. https://doi.org/10.1083/jcb.108.6.2401

HeadNeck.pdf. (n.d.). Retrieved March 6, 2021, from https://www.who.int/selection_medicines/committees/expert/20/applications/HeadNeck.pdf

Huang, C., Qiu, Z., Wang, L., Peng, Z., Jia, Z., Logsdon, C. D., Le, X., Wei, D., Huang, S., \& Xie, K. (2012). A novel FoxM1-caveolin signaling pathway promotes pancreatic cancer invasion and metastasis. Cancer Research, 72(3), 655-665. https://doi.org/10.1158/0008-5472.CAN-11-3102

Jensen, D. H., Hedback, N., Specht, L., Høgdall, E., Andersen, E., Therkildsen, M. H., Friis-Hansen, L., Norrild, B., \& von Buchwald, C. (2014). Human Papillomavirus in Head and Neck Squamous Cell Carcinoma of Unknown Primary Is a Common Event and a Strong Predictor of Survival. PLOS ONE, 9(11). https://doi.org/10.1371/journal.pone.0110456

Jung, A. C., Ray, A.-M., Ramolu, L., Macabre, C., Simon, F., Noulet, F., Blandin, A.-F., Renner, G., Lehmann, M., Choulier, L., Kessler, H., Abecassis, J., Dontenwill, M., \& Martin, S. (2015). Caveolin-1-negative head and neck squamous cell carcinoma primary tumors display increased epithelial to mesenchymal transition and prometastatic properties. Oncotarget, 6(39), 41884-41901.

Kanehisa, M., \& Goto, S. (2000). KEGG: Kyoto encyclopedia of genes and genomes. Nucleic Acids Research, 28(1), 27-30. https://doi.org/10.1093/nar/28.1.27

Kato, K., Hida, Y., Miyamoto, M., Hashida, H., Shinohara, T., Itoh, T., Okushiba, S., Kondo, S., \& Katoh, H. (2002). Overexpression of caveolin-1 in esophageal squamous cell carcinoma correlates with lymph node metastasis and pathologic stage. Cancer, 94(4), 929-933.

Kuriakose, M. A., Chen, W. T., He, Z. M., Sikora, A. G., Zhang, P., Zhang, Z. Y., Qiu, W. L., Hsu, D. F., McMunnCoffran, C., Brown, S. M., Elango, E. M., Delacure, M. D., \& Chen, F. A. (2004). Selection and validation of differentially expressed genes in head and neck cancer. Cellular and Molecular Life Sciences CMLS, 61(11), 1372-1383. https://doi.org/10.1007/s00018-004-4069-0

Longworth, M. S., Wilson, R., \& Laimins, L. A. (2005). HPV31 E7 facilitates replication by activating E2F2 transcription through its interaction with HDACs. The EMBO Journal, 24(10), 1821-1830. https://doi.org/10.1038/sj.emboj.7600651 
Masood, R., Hochstim, C., Cervenka, B., Zu, S., Baniwal, S. K., Patel, V., Kobielak, A., \& Sinha, U. K. (2013). A novel orthotopic mouse model of head and neck cancer and lymph node metastasis. Oncogenesis, 2(9), e68-e68. https://doi.org/10.1038/oncsis.2013.33

Masuelli, L., Budillon, A., Marzocchella, L., Mrozek, M.-A., Vitolo, D., Di Gennaro, E., Losito, S., Sale, P., Longo, F., Ionna, F., Lista, F., Muraro, R., Modesti, A., \& Bei, R. (2012). Caveolin-1 overexpression is associated with simultaneous abnormal expression of the E-cadherin/ $\alpha-\beta$ catenins complex and multiple ErbB receptors and with lymph nodes metastasis in head and neck squamous cell carcinomas. Journal of Cellular Physiology, 227(9), 3344-3353. https://doi.org/10.1002/jcp.24034

McKaig, R. G., Baric, R. S., \& Olshan, A. F. (1998). Human papillomavirus and head and neck cancer: Epidemiology and molecular biology. Head \& Neck, 20(3), 250-265. https://doi.org/10.1002/(sici)10970347(199805)20:3<250::aid-hed11>3.0.co;2-o

Nestl, A., Von Stein, O. D., Zatloukal, K., Thies, W. G., Herrlich, P., Hofmann, M., \& Sleeman, J. P. (2001). Gene expression patterns associated with the metastatic phenotype in rodent and human tumors. Cancer Research, 61(4), 1569-1577.

Nohata, N., Hanazawa, T., Kikkawa, N., Mutallip, M., Fujimura, L., Yoshino, H., Kawakami, K., Chiyomaru, T., Enokida, H., Nakagawa, M., Okamoto, Y., \& Seki, N. (2011). Caveolin-1 mediates tumor cell migration and invasion and its regulation by miR-133a in head and neck squamous cell carcinoma. International Journal of Oncology, 38(1), 209-217.

Nohata, N., Hanazawa, T., Kikkawa, N., Mutallip, M., Fuse, M., Chiyomaru, T., Kawakami, K., Enokida, H., Nakagawa, M., Okamato, Y., \& Seki, N. (2011). Abstract 132: MiR-133a as a tumor suppressive microRNA targeting multiple oncogenes in head neck squamous cell carcinoma. Cancer Research, 71(8 Supplement), 132-132. https://doi.org/10.1158/1538-7445.AM2011-132

Rothberg, K. G., Heuser, J. E., Donzell, W. C., Ying, Y.-S., Glenney, J. R., \& Anderson, R. G. W. (1992). Caveolin, a protein component of caveolae membrane coats. Cell, 68(4), 673-682. https://doi.org/10.1016/0092-8674(92)90143-Z

Shannon, P., Markiel, A., Ozier, O., Baliga, N. S., Wang, J. T., Ramage, D., Amin, N., Schwikowski, B., \& Ideker, T. (2003). Cytoscape: A Software Environment for Integrated Models of Biomolecular Interaction Networks. Genome Research, 13(11), 2498-2504. https://doi.org/10.1101/gr.1239303

Szklarczyk, D., Morris, J. H., Cook, H., Kuhn, M., Wyder, S., Simonovic, M., Santos, A., Doncheva, N. T., Roth, A., Bork, P., Jensen, L. J., \& von Mering, C. (2017). The STRING database in 2017: Quality-controlled protein-protein association networks, made broadly accessible. Nucleic Acids Research, 45(Database issue), D362-D368. https://doi.org/10.1093/nar/gkw937

Tse, E. Y. T., Ko, F. C. F., Tung, E. K. K., Chan, L. K., Lee, T. K. W., Ngan, E. S. W., Man, K., Wong, A. S. T., Ng, I. O.-L., \& Yam, J. W. P. (2012). Caveolin-1 overexpression is associated with hepatocellular carcinoma 
tumourigenesis and metastasis. The Journal of Pathology, 226(4), 645-653.

https://doi.org/10.1002/path.3957

Yang, G., Truong, L. D., Wheeler, T. M., \& Thompson, T. C. (1999). Caveolin-1 expression in clinically confined human prostate cancer: A novel prognostic marker. Cancer Research, 59(22), 5719-5723.

Zhang, H., Su, L., Müller, S., Tighiouart, M., Xu, Z., Zhang, X., Shin, H. J. C., Hunt, J., Sun, S.-Y., Shin, D. M., \& Chen, Z. (2008). Restoration of caveolin-1 expression suppresses growth and metastasis of head and neck squamous cell carcinoma. British Journal of Cancer, 99(10), 1684-1694.

https://doi.org/10.1038/sj.bjc.6604735

Zhao, X., Yu, G., Yu, X., Wang, J., \& Pan, X. (2016). Caveolin-1 is overexpressed in hypopharyngeal squamous cell carcinoma and correlates with clinical parameters. Oncology Letters, 12(4), 2371-2374. https://doi.org/10.3892/ol.2016.4963

\section{Figures}

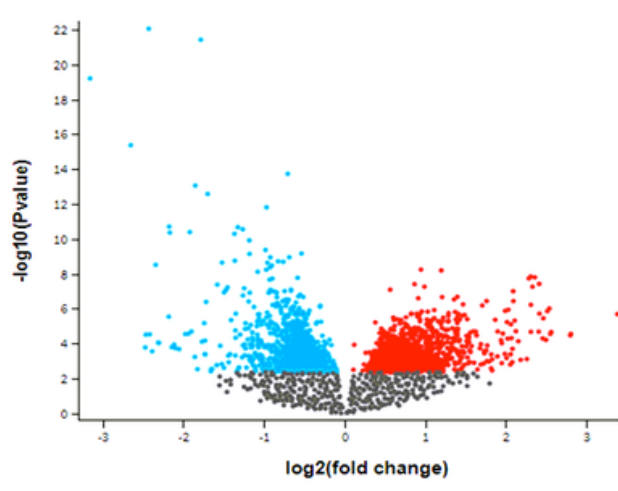

1(a)

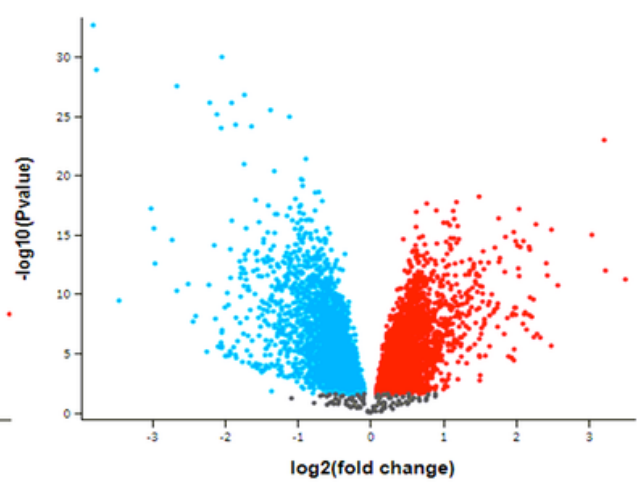

1(b)

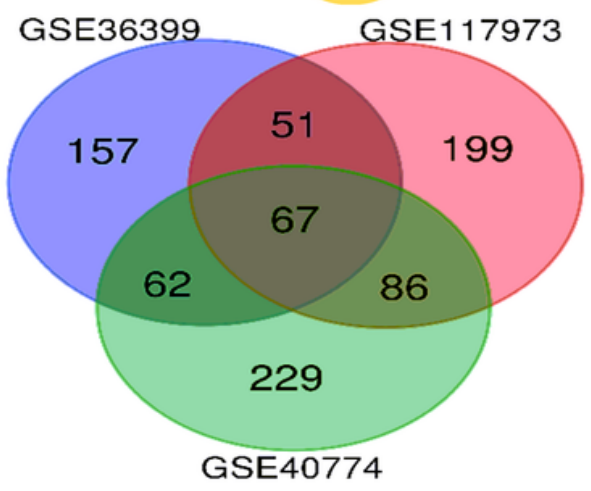

1(d)

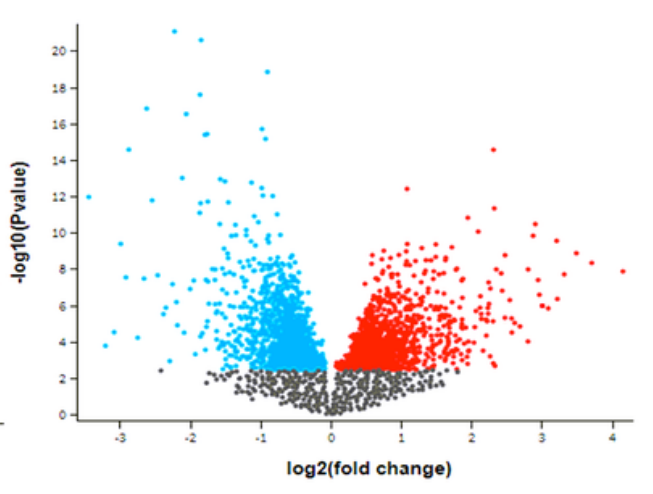

$1(c)$

\section{Figure 1}

(a-c) Volcano plots showing differentially expressed genes (d) Venn diagram representing the commonly identified DEGs. 


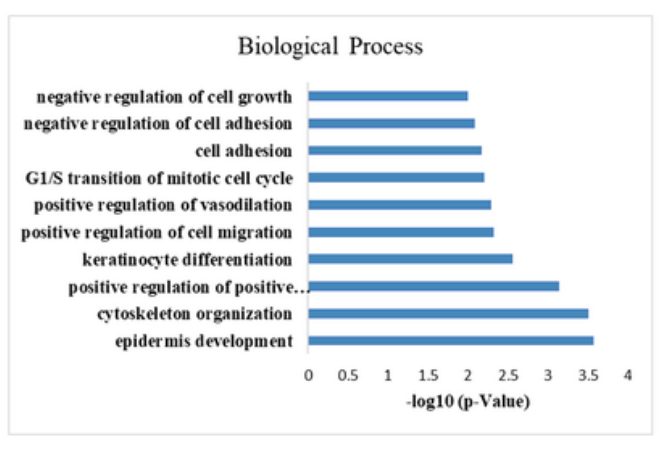

2(a)

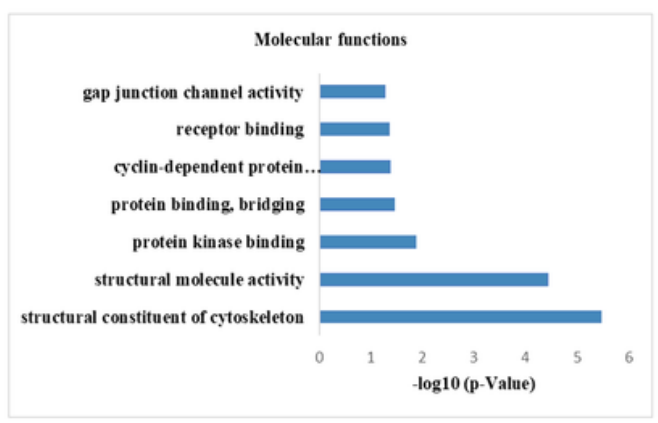

2(c)

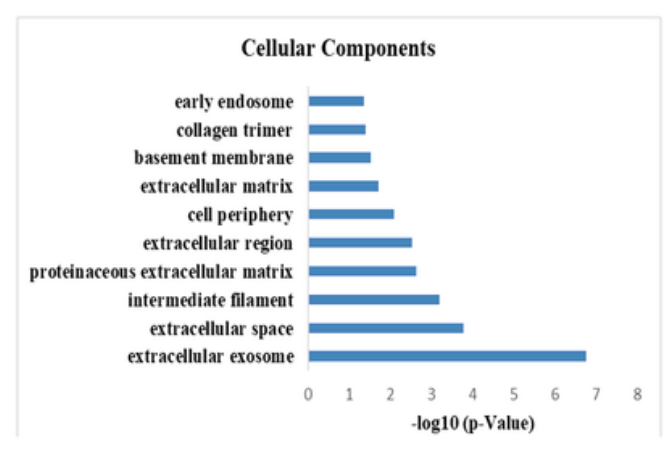

2(b)

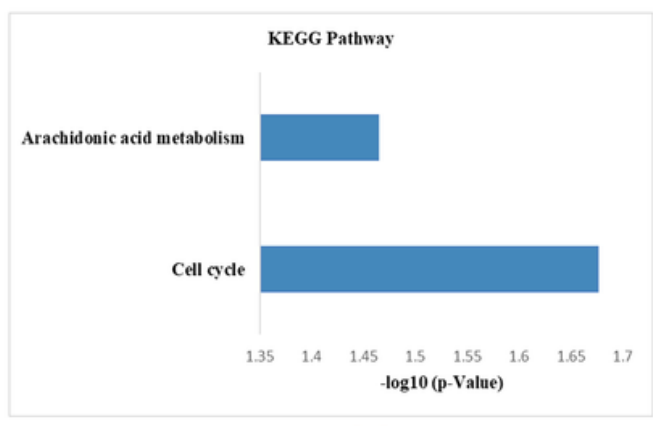

2(d)

Figure 2

KEGG pathway and GO terms identified for DEGs. 


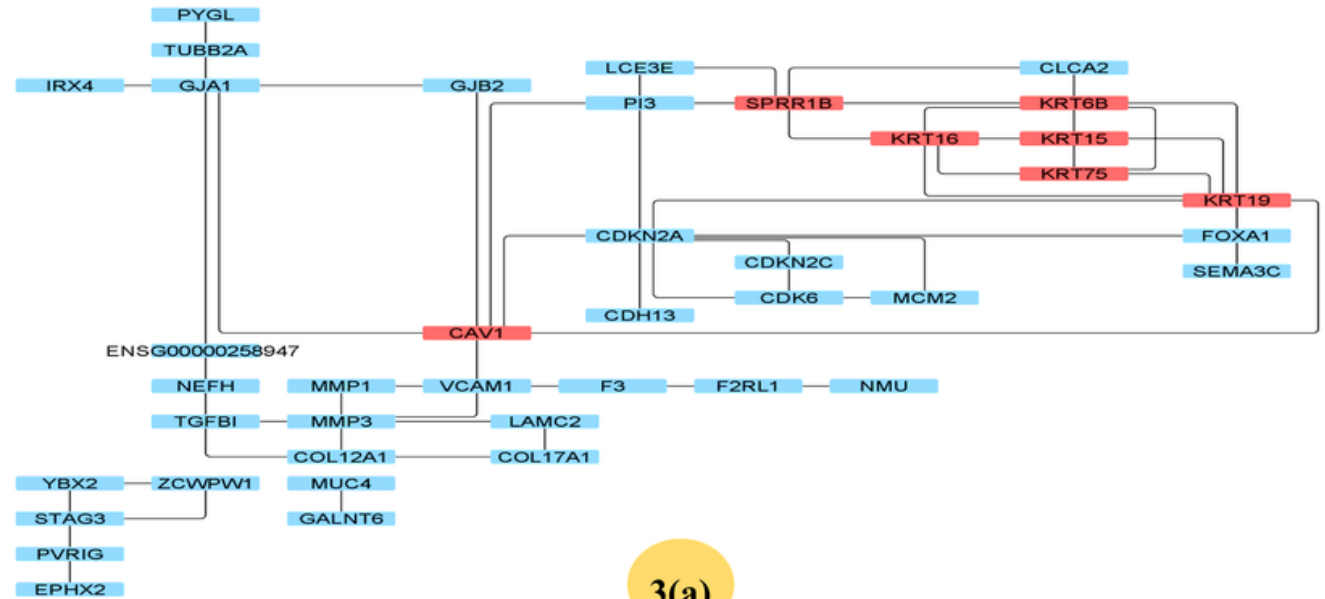

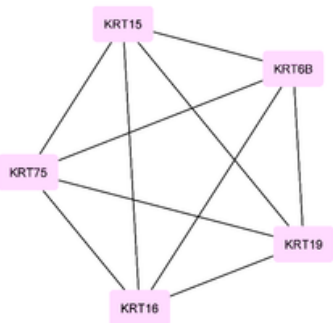

3(b)

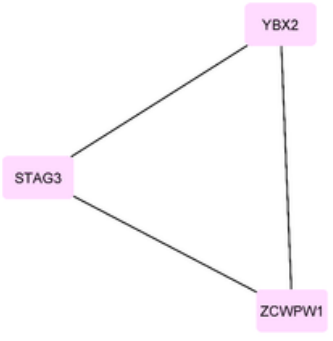

3(c)

\section{Figure 3}

(a) Protein-protein interaction network with highlighted common hub genes (b) Module1 and (c) Module2. 


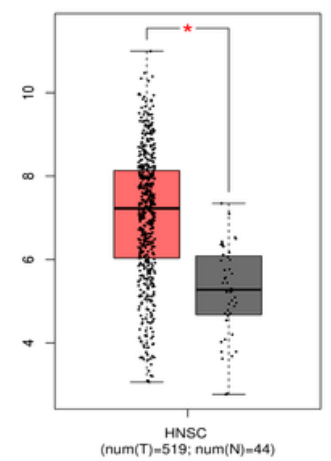

4(a)

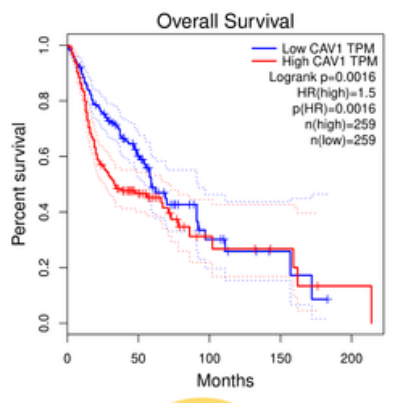

4(b)
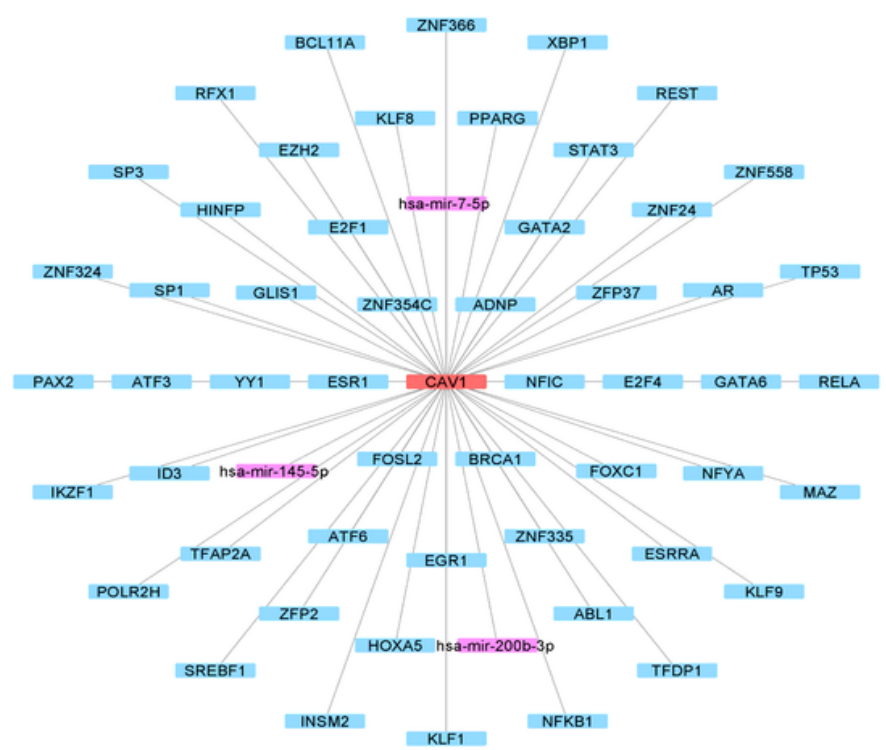

4(c)

\section{Figure 4}

(a) Differential expression of Cav1 in Head and neck cancer (b) Survival analysis curve for CAV1 gene (c) Hub gene targeting mi-RNA and transcription factors. 
Workflow

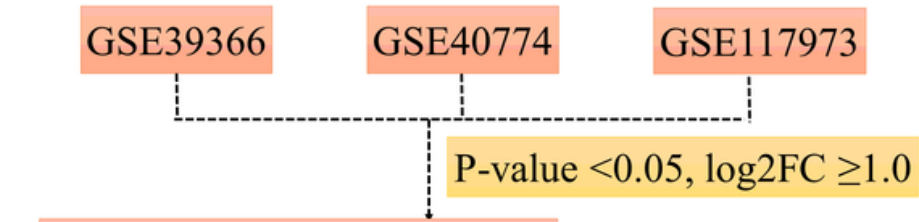

\section{Selection of significant genes}

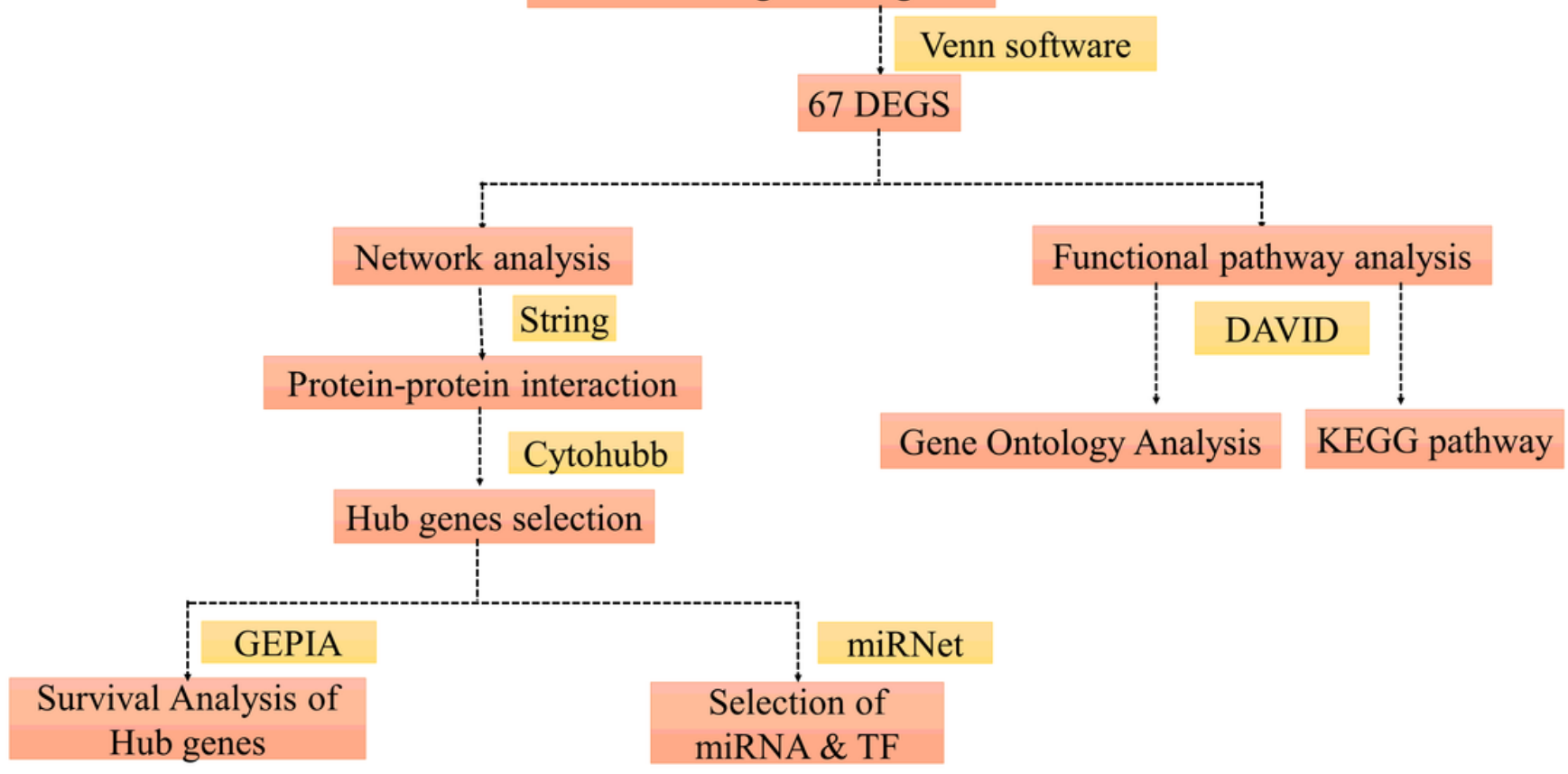

Figure 5

Workflow 